\title{
Sistema de almacenamiento de señales biológicas, con acceso remoto y parámetros de seguridad
}

\section{Biological signals storage system, with remote access and security parameters}

\author{
Nancy Esperanza Olarte López \\ Ingeniera en Telecomunicaciones. Asistente de Investigación, Grupo TIGUM de la Uni- \\ versidad Militar Nueva Granada. Bogotá, Colombia. \\ Contacto: nancy.olarte@unimilitar.edu.co

\section{Astrid Rubiano Fonseca} \\ Ingeniera en Mecatrónica, Magíster en Sistemas Automáticos de Producción. Docente \\ e Investigadora, Grupo TIGUM de la Universidad Militar Nueva Granada. Bogotá, Co- \\ lombia. Contacto: astrid.rubiano@unimilitar.edu.co
}

Palabras clave: acondicionamiento de señales, almacenamiento, autenticación, teleconsulta.

Key words: signal conditioning, storage, authentication, teleconsultation.

\section{RESUMEN}

El presente artículo muestra cómo se planteó el diseño e implementación de un sistema de almacenamiento de señales biológicas con parámetros de seguridad, manejando dos escenarios importantes: por un lado el tratamiento de señales y por otro el desarrollo de aplicaciones de software para llevar a cabo plataformas de telemedicina, lo anterior debido a que actualmente en los ambientes hospitalarios surge la necesidad de transmitir remotamente estas señales con la mejor calidad posible para que el especialista proporcione diagnósticos efectivos en el menor tiempo posible.
Dentro de su metodología, inicialmente fue propuesto el acondicionamiento de la señal ECG (electrocardiograma) y luego el tratamiento de la señal de temperatura. Actualmente se trabaja en la forma de onda de respiración para finalmente procesar la señal de presión sanguínea.

En el procesamiento de señales se propusieron diferentes cálculos para la implementación de circuitos con componentes electrónicos discretos y de superficie, el software planteado se creó bajo la plataforma Java, incluyendo parámetros de segu- 


\section{|investigación}

ridad como autenticación basada en conocimiento específico (nombre usuario, contraseña y respuesta a pregunta secreta), para luego una posterior visualización de varias señales ECG y temperatura asociadas a varios pacientes. La información adquirida es almacenada y gestionada de manera centralizada en el servidor del grupo de investigación en Telemedicina de la Universidad Militar Nueva Granada - TIGUM, donde se desarrolló el proyecto.

Adicionalmente el prototipo final permite incluir más protocolos, tecnologías de comunicación para la transmisión y recepción de datos, y parámetros de seguridad para la implementación de proyectos futuros.

\begin{abstract}
This paper shows the design and implementation of a storage system with security parameters intended for biological signals. The system deals with two important aspects. On the one hand, signal processing; on the other hand, software application developments that support telemedicine platforms. The rationale behind the implementation of such systems is the current situations in hospital environments, where there is the need to transmit these signals remotely with the best possible quality so as to provide an effective specialist diagnosis in the shortest time possible.
\end{abstract}

First, within this methodology, ECG conditioning signal was proposed; then, temperature signal processing was also proposed. Additionally, the project considers respiration waveforms, and blood pressure signals are also processed.

Different calculations were proposed for signal processing in order to implement circuits with discrete and surface electronic components. The implemented software was created using the Java platform, including security authentication parameters based on specific knowledge (username, password and a secret question answer). This allowed subsequent visualization of several ECG and temperature signals associated with patients. The information captured is centrally stored and managed at the Military University Telemedicine Research Group - TIGUM server - where the whole project was developed.

Additionally, the final prototype allows including more protocols, communication technologies for data transmission and reception, as well as security parameters for future implementation.

\section{INTRODUCCIÓN}

Actualmente la captura de medidas fisiológicas ha permitido tener un amplio desarrollo tecnológico, no solo para almacenarlas y estudiarlas posteriormente, sino también registrarlas e interpretarlas en tiempo real.

Esta evolución permite la transmisión a distancia de dichos datos, obteniendo resultados estadísticamente significativos en cuanto al seguimiento y supervisión de las condiciones del paciente como soporte a una estimación del posible diagnóstico por parte del especialista.
Para adquirir este tipo de señales, en lo posible se debe tratar de que la técnica a utilizar sea no invasiva y que posteriormente los datos fisiológicos sean procesados para su posterior análisis [1], permitiendo tener una visibilidad mucho más profunda a datos que son ocultos mediante técnicas tradicionales de valoración.

Sin embargo, aunque existen extensos estudios, diseños y desarrollos en el área del procesamiento de señales y el almacenamiento de datos biológicos, actualmente se tienen inconvenientes como son los parámetros de seguridad a implementar respecto a 


\section{investigación}

la información de los pacientes, los protocolos y la adecuada normatividad para el desarrollo de los sistemas remotos de consulta y la correspondiente visualización de las señales adquiridas.

\section{METODOLOGÍA}

Ya que actualmente las plataformas están conformadas por diferentes sistemas y servicios en telemedicina, la intención de este trabajo fue integrar varios de sus servicios, como es el tratamiento de las señales, su recepción, almacenamiento y visualización, considerando parámetros como acceso remoto y autenticación, con la finalidad de implementar un sistema que pueda ser utilizado por los médicos sin necesidad de hacer desplazamientos para generar diagnósticos a tiempo.

\subsection{Fases}

Esta se llevó a cabo mediante tres fases, las cuales se aprecian a continuación en la figura 1 :

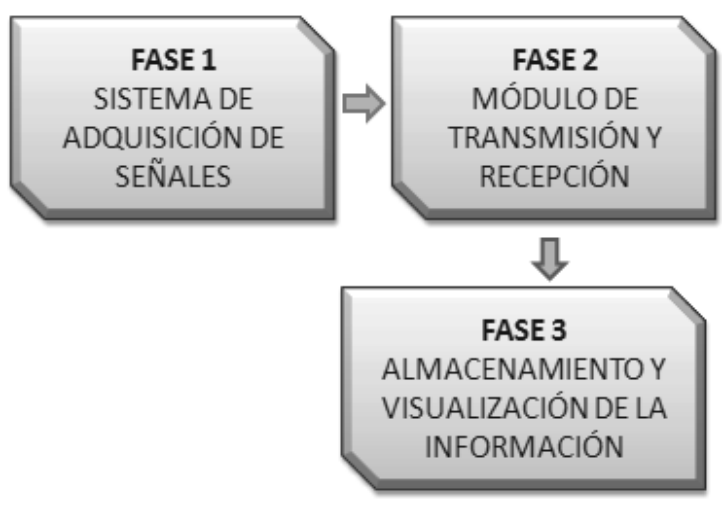

Figura 1. Metodología del proyecto

Fuente: elaboración propia.

\subsubsection{Fase 1: Sistema de adquisición de señales}

Inicialmente, en esta fase se incluyeron tres etapas dentro del diseño, las cuales se muestran en la figura 2 y se describen a continuación:

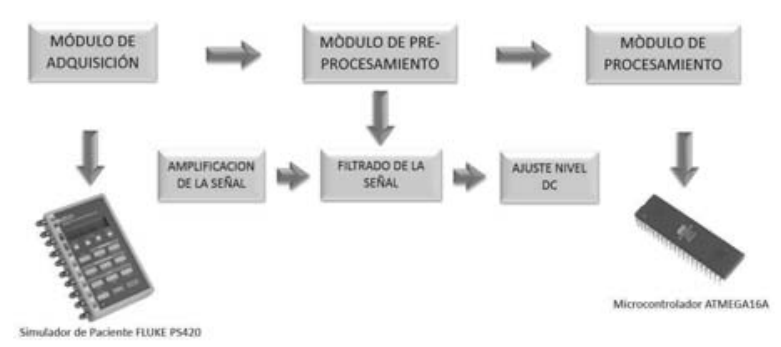

Figura 2. Fase 1: Sistema de adquisición de señales Fuente: elaboración propia.

\section{Módulo de adquisición}

En esta etapa se valoró y analizó a profundidad el estado del simulador depaciente Fluke PS420, el cual en este proyecto fue de gran importancia, ya que genera cuatro tipos de señales: ECG, temperatura, presión sanguínea y respiración. Este simulador de paciente se puede apreciar en la parte izquierda de la figura 2.

Inicialmente la señal escogida para su acondicionamiento fue la ECG por su alta trayectoria, análisis y documentación en el campo de la bioingeniería. Otro importante motivo es que el simulador reproduce este tipo de onda tanto en pacientes adultos como en pediátricos y 37 tipos diferentes de arritmias [2].

Para que el paciente PS420 genere este tipo de señal, se utilizan cables blindados llamados latiguillos, los cuales se observan en la figura 3. Estos vienen incluidos junto con el simulador de paciente y se conectan a las primeras tres derivaciones (RA, LAy RL) del paciente, correspondientes al brazo derecho, brazo izquierdo y pierna izquierda, de donde provienen las derivaciones estándares bipolares del triángulo de Einthoven para generar la onda cardiaca [3]. Al conectar estos latiguillos (figura 3), el paciente se introduce el código correspondiente a la señal ECG. 


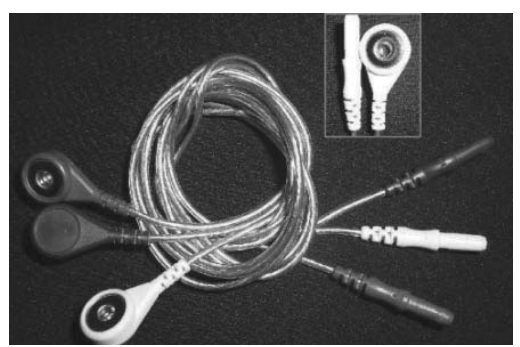

Figura 3. Latiguillos ECG [4]

Fuente: tomada de[7].

\section{Módulo de pre-procesamiento}

Luego de generar la señal ECG, esta fase se dividió en tres etapas: amplificación de la señal, filtrado y ajuste de nivel DC, tal y como se muestra en la figura 2 (en la mitad). Este módulo fue de gran importancia, ya que es el encargado de interpretar y acondicionar las señales provenientes del paciente, para que posteriormente sean procesadas.

Inicialmente para la amplificación de la señal ECG, fue utilizado el amplificador de instrumentación AD620 por su estabilidad y amplio uso en electrocardiografia, ya que posee una ganancia entre 1 y 1000 y una alimentación entre 2,3V y $18 \mathrm{~V}$. También dentro de los componentes de esta etapa se utilizó el amplificador operacional JFET TL084CN por su bajo consumo de potencia y bajo ruido.

Luego, la etapa de filtrado elimina el ruido indeseado proveniente de fuentes externas como la red eléctrica, el contacto de los latiguillos con los circuitos y radio frecuencia. El filtro implementado en el diseño fue un filtro pasabanda, el cual limitó el espectro de frecuencias de la señal ECG entre $0,5 \mathrm{~Hz}$ y $150 \mathrm{~Hz}$.

Por último, fue necesario ajustar el nivel DC de la señal ECG, ya que las señales provenientes de la etapa de filtrado se encontraban en un rango de tensión negativo, y en la siguiente etapa es necesario trabajar con valores de tensión positivos.

Para el acondicionamiento de la señal de temperatu-

\section{investigación}

ra, esta se obtuvo mediante el criterio de selección de la carga de prueba, ya que el simulador de paciente utilizado reproduce las condiciones normales hipotérmicas e hipertérmicas con cinco valores preestablecidos de temperatura [5], tal y como se muestra en la tabla 1, la cual indica el código a ingresar correspondiente a cada temperatura.

Tabla 1. Código de temperatura y valores correspondientes.

\begin{tabular}{|c|c|}
\hline $\begin{array}{c}\text { Código del Paciente } \\
\text { PS 420 }\end{array}$ & Temperatura \\
\hline 185 & I. $30^{\circ} \mathrm{C}\left(86^{\circ} \mathrm{F}\right)$ \\
\hline 186 & II. $35^{\circ} \mathrm{C}\left(95^{\circ} \mathrm{F}\right)$ \\
\hline 187 & III. $37^{\circ} \mathrm{C}\left(98,6^{\circ} \mathrm{F}\right)$ \\
\hline 188 & IV. $40^{\circ} \mathrm{C}\left(104^{\circ} \mathrm{F}\right)$ \\
\hline 189 & V. $42^{\circ} \mathrm{C}\left(107,6^{\circ} \mathrm{F}\right)$ \\
\hline
\end{tabular}

Fuente: tomada de [2].

Además, fue necesario utilizar el cable con conector Mini Din de ocho pines [6], tal y como se muestra en la figura 4, ya que la salida de los datos correspondientes a la señal de temperatura provenientes del paciente utiliza este tipo de conector.

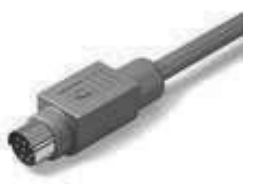

Figura 4. Cable con conector Mini Din ocho pines Fuente: tomada de [6].

La distribución de los ocho pines del conector Mini Din hembra del paciente [7], se muestra a continuación en la figura 5: 


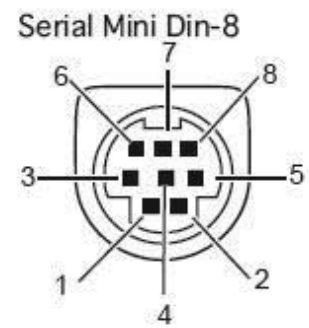

Figura 5. Distribución de pines conector Mini Din Fuente: tomada de [7].

Como el cable utilizado para el paciente, tiene los 8 hilos diferenciados por colores, para los ocho pines del conector, en la tabla 2, se observa la distribución y función de cada uno de ellos.

Tabla 2. Distribución de pines Mini Din por colores de cable.

\begin{tabular}{|c|c|c|}
\hline Pin & Color cable & Descripción \\
\hline 1 & Negro & $\begin{array}{c}\text { Secondary output (5-12V DC } \\
\text { default configuration) }\end{array}$ \\
\hline 2 & Café & $\begin{array}{c}\text { Primary 4-20mAcurrent } \\
\text { output }\end{array}$ \\
\hline 3 & Rojo & RS232 serial input \\
\hline 4 & Naranja & Set pointintput/remote \\
\hline 5 & Amarillo & RS232 serial output \\
\hline 6 & Verde & $\begin{array}{c}\text { Primary DC output } \\
(0-5 V \text { default })\end{array}$ \\
\hline 7 & Azul & DC Power input \\
\hline 8 & Púrpura & Ground \\
\hline
\end{tabular}

Fuente: tomada de [6].

Se utilizó en esta etapa el Teorema de Máxima Transferencia de Potencia, logrando interpretar los datos provenientes del paciente para luego procesarlos en la interfaz eléctrica.

\section{Módulo de procesamiento}

Este módulo convirtió los datos análogos a digitales. Para esto se programó el microcontrolador ATMEGA16A de 40 pines de la familia AVR de ATMEL, el cual se aprecia en la figura 2 (parte derecha).
Este permite la entrada hasta de ocho señales biométricas, por sus ocho canales de conversión Análogo/Digital de 10 bits, memoria flash de 16 Kbytes, 512 bytes de memoria EEPROM y USART serial programable. Para procesar los datos se utilizó una frecuencia de muestreo de $300 \mathrm{~Hz}$, la cual es el doble de la frecuencia de corte por teorema de Nyquist.

\subsubsection{Fase 2: Módulo de transmisión y recepción}

En la segunda fase se diseñó e implementó el Módulo de Transmisión y Recepción, seleccionando el estándar RS232 para la comunicación de los datos hacia el computador. Para lograr esto, se implementó el circuito Max232 para la conversión de niveles TTL $(0$ a $5 \mathrm{~V})$ del microcontrolador a niveles RS232 $(12 \mathrm{a}-12 \mathrm{~V})$, junto con la utilización del conector DB9 (figura 6) hacia el puerto serial del computador. Por último, se enviaron los datos de la señal ECG en caracteres ASCII en Hyperterminal, para su posterior conversión en decimal para su gráfica y almacenamiento en la base de datos.

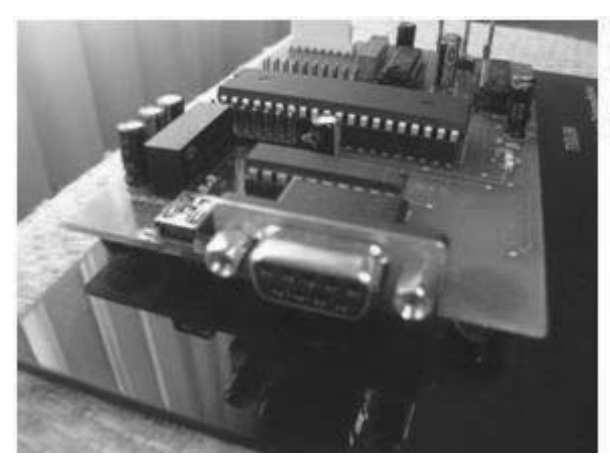

Figura 6. Conector DB9 para comunicación RS-232 Fuente: elaboración propia.

\subsubsection{Fase 3: Almacenamiento y visualización de la información}

Para esta última fase se implementó bajo la plataforma JAVA un software diseñado para trabajar en un entorno web con el fin de facilitar el acceso a la información desde el lugar en el que se encuentre el usuario necesitando solamente de un equipo con 


\section{|investigación}

acceso a internet y un navegador web. Su diseño permite visualizar varias señales ECG a la vez y diferentes registros de temperatura, previo almacenamiento de estas, para la futura valoración del médico especialista.

Para el desarrollo de esta fase fue importante tener en cuenta la información de cada paciente. Para lo anterior se creó una base de datos en PostgreSQL, incluyendo la información personal y de contacto de los médicos y los pacientes mediante el diligenciamiento de un formulario.

También se identificaron los posibles ataques de información que se podrían presentar sobre el sistema, producto de esto se diseñaron e implementaron los parámetros de seguridad para proteger la información y como consecuencia denegar su acceso.

A causa de esto y por tratarse de un sistema centralizado, el acceso a la información se efectuó mediante autenticación ante un servidor basado en conocimiento específico, es decir, con un nombre de usuario, clave y la respuesta a una pregunta secreta.

Luego de reconocer a cada usuario y clasificándolo dentro de un perfil de trabajo definido previamente por el administrador del sistema, se definen dos usuarios identificables: operario y medico.

- Operario: está encargado únicamente de tomar el examen, vincularlo a un paciente y almacenarlo en la base de datos.

- Médico: tiene acceso a la información de los pacientes incluyendo los exámenes, y puede realizar diagnósticos de cada uno.

La señal ECG, los registros de temperatura adquiridos junto con la información del personal médico y los pacientes, es almacenada y gestionada de manera centralizada en el servidor del grupo de investigación, facilitando de esta manera su administración y control.
Las etapas de esta fase se resumen en la figura 7. Inicialmente se tiene una etapa de autenticación y se identifican los dos perfiles de usuario, posteriormente se presentan los tipos de consulta, luego la información médica, los exámenes asociados a los pacientes y por último, la visualización de los exámenes.

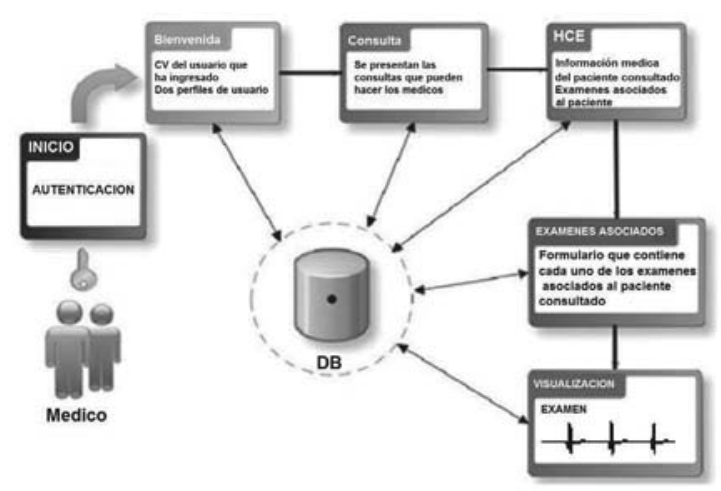

Figura 7. Etapas de almacenamiento y gestión centralizada de la información.

Fuente: elaboración propia.

\section{RESULTADOS}

Para acondicionar la señal ECG, se implementaron en protoboard alimentando con fuente dual regulada de $5 \mathrm{Vy}-5 \mathrm{~V}$, las pruebas de amplificación, comparación de eliminación de ruido indeseado y ajuste de nivel DC, lo anterior para el procesamiento de datos y futura transmisión de estos hacia el computador. A continuación se describirán los resultados.

Ya que los rangos de voltaje apropiados para las señales de ECG se encuentran entre $1 \mathrm{mV}$ y $10 \mathrm{mV}$, el primer elemento seguido conectado al paciente Fluke PS420 por medio de los electrodos fue la etapa de amplificación, por medio del AD620, el cual permitió que esta señal se amplificara hasta 100 veces más, atenuando a su vez componentes en modo común. El circuito esquemático de esta etapa se puede apreciar en la figura 8. 


\section{investigación |}

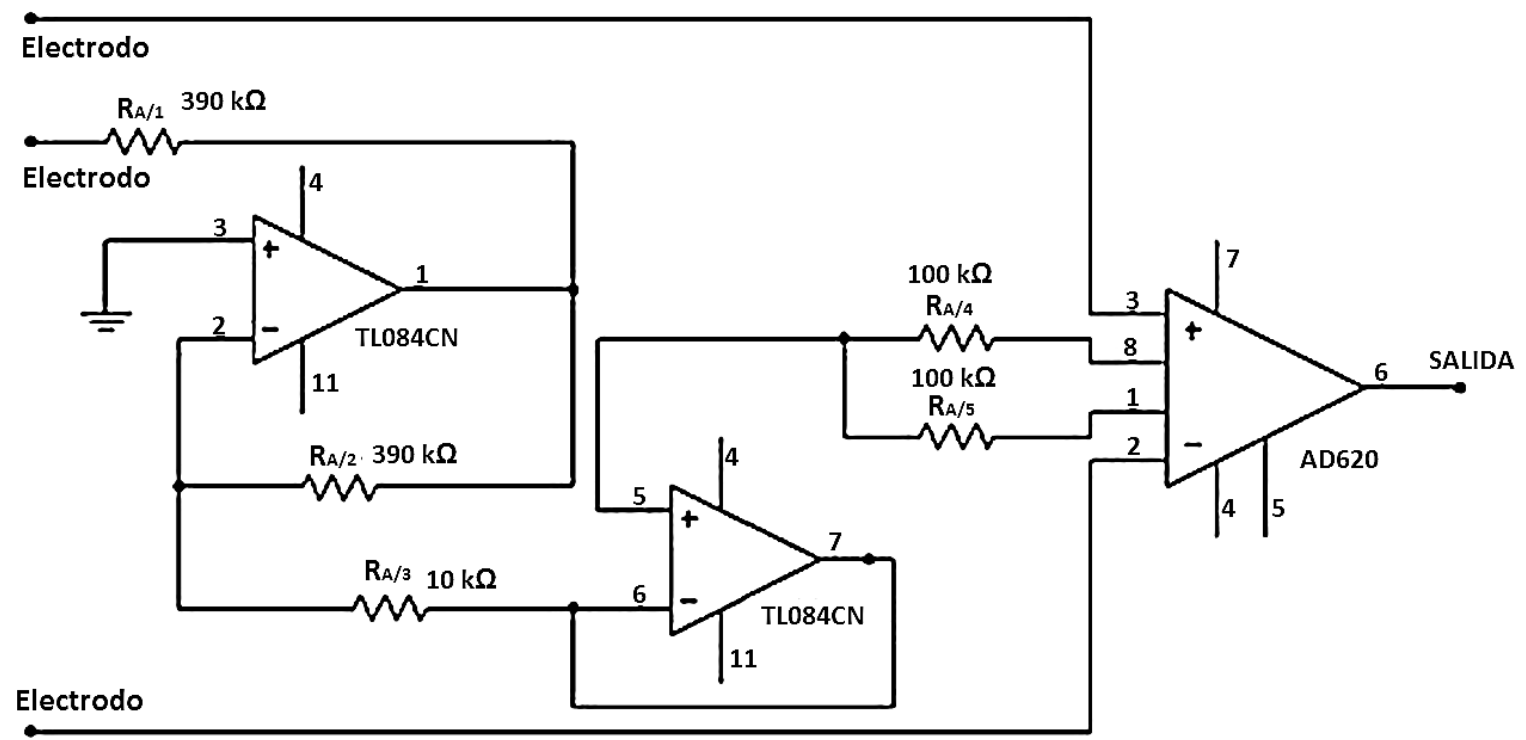

Figura 8. Etapa amplificación.

Fuente: elaboración propia.

Luego se realizaron cálculos del filtro pasabanda, para eliminar componentes de alta y baja frecuencia e interferencias de $60 \mathrm{~Hz}$, para esto se implementó un filtro pasa alto de $40 \mathrm{~dB} /$ década (figura 9) en cascada con un filtro paso bajo de $-60 \mathrm{~dB} /$ década (figura 10).

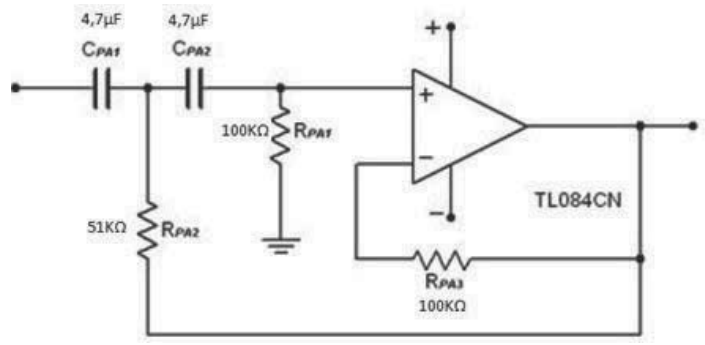

Figura 9. Filtro pasa alto.

Fuente: elaboración propia.

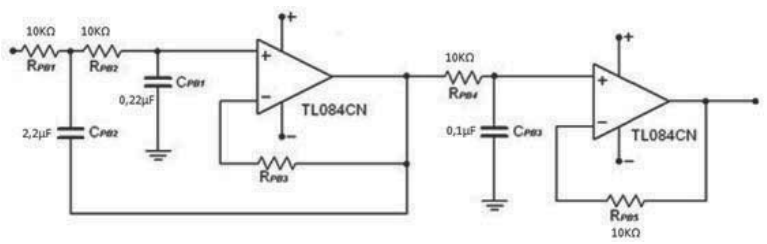

Figura 10. Filtro pasa bajo. Fuente: elaboración propia.
La onda cardiaca generada con ruido y sin él se puede apreciar en la figura 11.

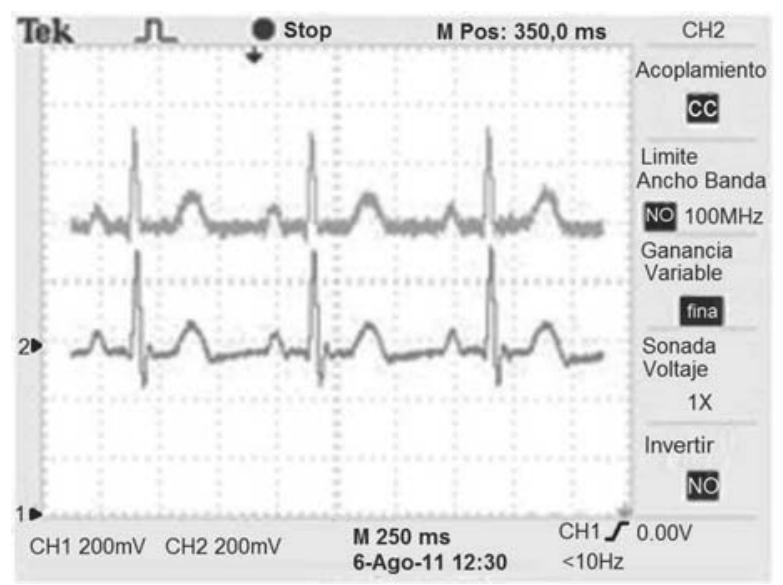

Figura 11. Comparación señal ECG sin filtrar y filtrada. Fuente: elaboración propia.

Posteriormente, como las señales provenientes de la etapa de filtrado se encuentran en un rango de $-1 \mathrm{~V}$ a $3 \mathrm{~V}$, se diseñó un circuito para ajustar el nivel DC con el objetivo de llevar la señal a un intervalo de 
tensión entre $0 \mathrm{~V}$ y $5 \mathrm{~V}$, ya que en la siguiente etapa, el microprocesador trabaja solamente con valores de tensión positivos. El circuito esquemático de esta etapa se muestra en la figura 12.

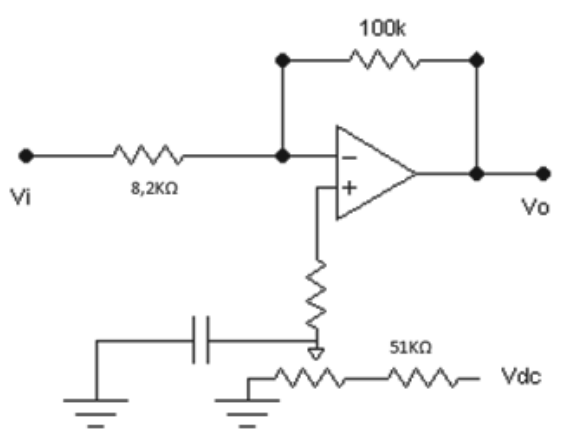

Figura 12. Circuito para ajuste de nivel DC Fuente: elaboración propia.

El resultado de la última etapa de filtrado, junto con el ajuste de nivel DC, se muestra a continuación en la figura13. Como se puede apreciar, la señal ECG después de este ajuste está inversa, pero, aunque cumple con los intervalos de tensión positivos, fue necesario en la etapa de procesamiento programar el microcontrolador para que la señal fuera invertida nuevamente y así transmitir estos datos hacia el computador para finalmente visualizarla en pantalla.

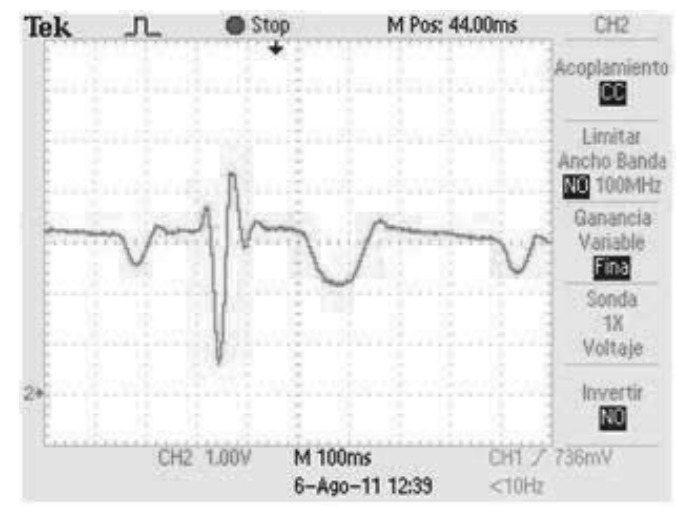

Figura 13. Señal ECG con ajuste de nivel DC Fuente: elaboración propia.
Asimismo, para el acondicionamiento de los datos de temperatura, se realizaron mediciones de las impedancias de salida correspondientes a las diferentes opciones de temperatura del paciente, como se muestra en la tabla 3. Como resultado se determinó que el valor medio fue de $1461,5 \Omega$, aproximandose este valor a una impedancia promedio de $1,5 \mathrm{~K} \Omega$, el cual ajusta la carga de prueba de forma tal que garantiza el cumplimiento del teorema de máxima transferencia de potencia para el acondicionamiento de estos datos.

Tabla 3. Valores de temperatura junto con los resultados de impedancia.

\begin{tabular}{|c|c|}
\hline Temperatura & Impedancia $(\boldsymbol{\Omega})$ \\
\hline VI. $30^{\circ} \mathrm{C}\left(86^{\circ} \mathrm{F}\right)$ & 1815 \\
\hline VII. $35^{\circ} \mathrm{C}\left(95^{\circ} \mathrm{F}\right)$ & 1471 \\
\hline VIII. $37^{\circ} \mathrm{C}\left(98,6^{\circ} \mathrm{F}\right)$ & 1355 \\
\hline IX. $40^{\circ} \mathrm{C}\left(104^{\circ} \mathrm{F}\right)$ & 1200 \\
\hline X. $42^{\circ} \mathrm{C}\left(107,6^{\circ} \mathrm{F}\right)$ & 1108 \\
\hline
\end{tabular}

Fuente: elaboración propia.

Para la implementación del circuito impreso, inicialmente se realizaron los diseños en el software Eagle. Se tuvieron en cuenta dos capas, en la primera se tienen componentes discretos y en la segunda componentes de superficie como por ejemplo las resistencias junto con todos sus recorridos.

Como resultado final, los datos provenientes del simulador Paciente PS420 Fluke tanto para la onda cardiaca ECG como para temperatura se ubicaron en la parte derecha del circuito impreso tal y como se muestra en la figura 14 . 


\section{investigación}

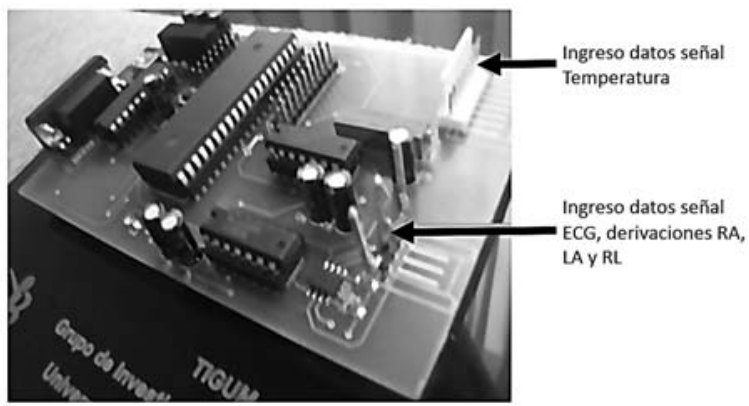

Figura 14. Circuito con entradas señales ECG y temperatura. Fuente: elaboración propia.

Por último, el prototipo final se puede apreciar en la figura 15. La etapa de alimentación de los $5 \mathrm{~V}$ proviene por USB, luego el regulador de voltaje deriva $-5 y+5 \mathrm{~V}$, seguido de las etapas de acondicionamiento, procesamiento de señales y transmisión y recepción por RS-232 hacia el PC.

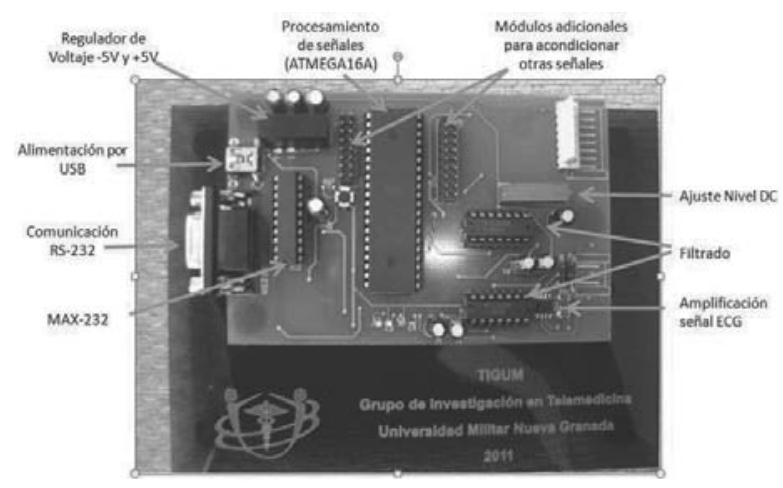

Figura 15. Circuito impreso final.

Fuente: elaboración propia.

\section{CONCLUSIONES}

El criterio de selección utilizado para acondicionar los datos correspondientes al cambio de temperatura, permitió tener una mayor variación de voltaje en la carga de prueba ante los diferentes cambios de los parámetros de temperatura provenientes del simulador.
El prototipo final del circuito impreso se realizó en lo posible con el menor tamaño $(8 \mathrm{~cm} \times 12 \mathrm{~cm})$ y mejor portabilidad, ya que para su alimentación no se necesitan fuentes duales externas, por su alimentación por puerto USB y su regulador de voltaje que deriva $-5 \mathrm{~V}$ y $+5 \mathrm{~V}$.

Igualmente, este prototipo permite también agregar más circuitos impresos como lo pueden ser el circuito para el acondicionamiento de señal de respiración y presión sanguínea, señales que también genera el simulador de paciente Fluke PS420, y en las que actualmente se viene trabajando para su acondicionamiento.

También existe la posibilidad de agregar más protocolos y tecnologías de comunicación para transmitir y recibir datos, aparte del RS-232. Para proyectos futuros se desea implementar más parámetros de seguridad, o utilizar también otras plataformas o lenguajes de programación.

\section{FINANCIAMIENTO}

Este proyecto de investigación, perteneciente al grupo TIGUM (Grupo de Investigación en Telemedicina de la Universidad Militar), fue presentado a convocatoria a finales del año 2010 y aprobado para su financiamiento, ejecución y representación en eventos nacionales e internacionales durante el año 2011, por parte de la Vicerrectoría de Investigaciones de la misma universidad.

\section{AGRADECIMIENTOS}

A la Universidad Militar Nueva Granada por la financiación del proyecto "Sistema de almacenamiento de señales biológicas, con acceso remoto y parámetros de seguridad - ING 727". 


\section{investigación}

\section{REFERENCIAS}

[1] Jurik, A. Biotelemetry and Body Sensors: Enabling ECG Monitoring, Charlottesville, Virginia: University of Virginia, 2009, pp. 2-4.

[2] Fluke Corporation,Fluke Biomedical PS420 Multiparameter Simulator: Manual de uso, 2006 - 2007, Estados Unidos: Fluke Corporation, p. 21.

[3] A. Rubiano F., N. Olarte and D. Lara, Design of Medical Information Storage System - ECG Signal, in World Academy of Science, Engineering and Technology 59, 2011, p. 901.

[4] L.López, Tecnología ZigBee aplicada a la telemetría de bioseñales para redes inalám- bricas de área personal. Bogotá: Universidad Militar Nueva Granada,2010, p. 63.

[5] Fluke Corporation, Fluke Biomedical PS420 Multiparameter Simulator: Manual de Uso, 2006 - 2007, Estados Unidos: Fluke Corporation, p. 16.

[6] C2G Lastar, Cable con conector mini din 8 pines. [en línea]. Disponible (21 de febrero de 2012): http://www.cablestogo.com/resources/din.asp

[7] Leroy Davis, Distribución de pines conector mini din. [en línea]. Disponible (26 de febrero de 2012): http://www.interfacebus. com/Apple_MiniDIN_PinOut.html. 